PAPER

\title{
Ultrasonic position and velocity measurements for a moving object by the simultaneous transmission of preferred-pair M-sequences
}

\author{
Shinnosuke Hirata*,†, Kota Yamanaka and Hiroyuki Hachiya \\ Department of Systems and Control Engineering, School of Engineering, Tokyo Institute \\ of Technology, 2-12-1 Ookayama, Meguro-ku, Tokyo, 152-8550 Japan
}

(Received 11 October 2019, Accepted for publication 12 February 2020)

\begin{abstract}
A method of ultrasonic position and velocity measurements for a moving object by Msequence pulse compression has been proposed. In the proposed method, the Doppler velocity is estimated from the peak in the autocorrelation function of the received signal, which includes cyclic $\mathrm{M}$-sequences reflected from the object. Then, the received signal is correlated with the reference Msequence expanded or compressed in accordance with the estimated Doppler velocity. The position is determined from the acoustic image formed from cross-correlation functions obtained in different propagation paths of ultrasound. In this paper, the simultaneous transmission of preferred-pair Msequences is studied to improve the spatial resolution of the image while keeping the original frame rate of the proposed method. Experiments using the moving pole, two loudspeakers and three microphones are conducted to evaluate the estimated Doppler velocities and determined positions. In most situations, Doppler velocities can be accurately estimated when two different Doppler components are included in the received signal. In some situations, however, accurate Doppler velocities inevitably cannot be estimated due to the peak overlapping in the autocorrelation function. The accuracy of determined positions may degrade when the signal-to-noise ratio of the received signal is insufficient or Doppler velocities are not accurately estimated.
\end{abstract}

Keywords: Pulse compression, M-sequence, Doppler velocity estimation, Acoustic image

PACS number: 43.20.Ye, 43.35.Yb [doi:10.1250/ast.41.857]

\section{INTRODUCTION}

Ultrasonic sensors are widely used to acquire target information or medium information, for nondestructive testing. Measurement using ultrasonic sensors has several advantages: low purchase cost, small size, and simple structure. In particular, ultrasonic measurement is expected to be applied in autonomous driving technology, together with millimeter-wave radar and camera images [1-4]. In this technology, the distance to the target and the velocity of the object are the most important information to be measured.

The pulse-echo method is one of the distance measurement methods using ultrasound. In this method, an ultrasonic pulse is transmitted and the propagation time of its echo reflected from an object is determined. Then, the pulse (echo) propagation distance can be estimated from the time of flight (TOF) and the speed of sound in the

*e-mail: shin@chiba-u.jp

${ }^{\dagger}$ Present affiliation: Center for Frontier Medical Engineering, Chiba University, 1-33 Yayoi-cho, Inage-ku, Chiba, 263-8522 Japan medium. The distance resolution of the pulse-echo method is associated with the pulse width, which predominantly depends on bandwidths of ultrasonic transducers. In the case of a short pulse, however, environmental noise or read-out noise make it difficult to identify the echo.

To improve the signal-to-noise ratio (SNR) of the received signal and the resolution of the TOF, pulse compression has been introduced to the pulse-echo method [4-17]. A long burst with a sharp autocorrelation property, which is modulated by a frequency sweep (FS) or coded by pseudo-random numbers or noise (PN), is typically transmitted in pulse compression. Then, the received signal is correlated with the reference signal, which corresponds to the transmitted signal. In the cross-correlation function, the power of the burst is compressed into a pulse when the received signal and the reference signal match. Therefore, the TOF can be determined from the peak (the compressed pulse) in the cross-correlation function with high resolution.

In FS modulation, linear-frequency modulation or linear-period modulation is employed to modulate the transmitted signal [5-9]. The resolution of the compressed 
pulse is determined by the sweep bandwidth. The SNR improvement is determined by the bandwidth and the signal duration. In PN coding, a short pulse or its inverse pulse is assigned to each binary character [4,10-17]. When the reference signal is composed of discrete binary characters, the compressed pulse corresponds to the accumulated short pulses. Therefore, the resolution is determined by the short pulse width, and the degree of improvement of the SNR is determined by the number of binary characters.

In the case of a moving transducer or object, the transmitted signal is expanded or compressed owing to the Doppler effect. FS-modulated signals are relatively tolerant of Doppler shift [6-9]. On the other hand, Dopplershifted PN-coded signals cannot be correlated with original reference signals. However, a method of distance and Doppler velocity measurement for a moving object using an $\mathrm{M}$-sequence-coded signal has been studied. The $\mathrm{M}$ sequence is one of the pseudo-random binary numbers generated from a linear feedback shift register (LFSR). In this method, the Doppler velocity of the object is measured from the autocorrelation function of the received signal including the cyclic M-sequence-coded signal [16]. Then, the received signal is correlated with the Doppler-shifted reference signal, which is expanded or compressed in accordance with the measured Doppler velocity [17].

To measure the position of an object, different-path distance measurements are required. Particularly for an object smaller than the wavelength of ultrasound, the acoustic image can be formed from received signals along different paths by the synthesis aperture focusing technique [18]. The spatial resolution of the acoustic image is determined by the aperture width, which is the distance between the farthest transducers in different paths. The SNR improvement is determined by the number of paths. Furthermore, to improve the resolution and the SNR within the restricted size of the measurement system, multichannel transmission and multichannel reception are effective. In the case of the simultaneous transmission of multichannel ultrasound, however, transmitted signals interfere with each other. In PN coding, multichannel transmitted signals can be easily generated by the employment of different codes or modulations with low interference $[19,20]$.

In previous works, positions and velocities of a moving object measured by the proposed method of M-sequence pulse compression using one loudspeaker and four microphones were evaluated [17]. In the present work, the employment of two loudspeakers and three microphones for improvement of the spatial resolution and the SNR of the acoustic image is studied. In this measurement system, multichannel transmitted signals coded by different $\mathrm{M}$ sequences with low interference are simultaneously transmitted to keep the high frame rate of acoustic images.
However, the interchannel interference has the potential to degrade the accuracy of positions or velocities. Furthermore, interference can occur also in the case of multiple objects. In particular, there is a serious concern about the effect of interference on Doppler velocity estimation. In the proposed method of M-sequence pulse compression, the received $\mathrm{M}$-sequence-coded signal cannot correlate with the Doppler-shifted reference signal in the case of incorrect Doppler velocity estimation. In this work, therefore, positions and velocities measured by the simultaneous transmission of preferred-pair M-sequences are evaluated with respect to the effect of M-sequence interference in two-dimensional experiments.

\section{METHOD}

\subsection{Position and Velocity Measurement Using Pre- ferred-pair M-sequences}

An M-sequence is a cyclic binary sequence generated from an LFSR. The order $n$ of the M-sequence is the length of the LFSR. The number $L$ of binary characters in one cycle of the M-sequence is $2^{n}-1$. In the cross-correlation function between a cyclic M-sequence and one cycle of the M-sequence, the correlation values are $L$ when both characters match, otherwise, the correlation values are -1 . Furthermore, white Gaussian noise included in the cyclic M-sequence is increased by $\sqrt{L}$ times. Therefore, the SNR in the cross-correlation function is improved by $\sqrt{L}$ times. If the number or positions of feedback taps in the LFSR are different, a different $\mathrm{M}$-sequence is generated. The preferred pair is the pair of different M-sequences whose correlation values are low and composed of only three values.

In this study, 11th-order M-sequences are employed to code ultrasound. $L$ and SNR improvement of the Msequence are 2,047 and $33.1 \mathrm{~dB}$, respectively. The preferred pair of $\mathrm{M}$-sequences $\mathrm{A}$ and $\mathrm{B}$ are selected, then, a sine wave and its inverse are assigned as +1 and -1 in the $\mathrm{M}$-sequence, respectively. The reference signal is one cycle of the M-sequence-coded signal. Examples of correlation functions of sine waves modulated by preferred-pair Msequences A and B are illustrated in Fig. 1. The amplitude on the ordinate is normalized by the correlation peak and the time on the abscissa is normalized by the length of the reference signal. Figure 1(a) shows the autocorrelation function of the cyclic M-sequence-coded signal A and the reference signal $\mathrm{A}$. The correlation values, except for the peak, are $-1 / 2,047$. Figure 1 (b) shows the cross-correlation function of the cyclic M-sequence-coded signal B and the reference signal A. The correlation values are 63/ $2,047,-1 / 2,047$, and $-65 / 2,047$. In Fig. 1(c), cyclic Msequence-coded signals $\mathrm{A}$ and $\mathrm{B}$ are summed with a time difference of 0.006. Then, the summed signal is correlated with each reference signal A or B. In the case of an 11th- 


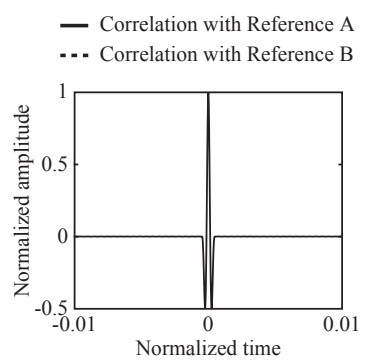

(a) Transmission of $\mathrm{M}$-sequence $\mathrm{A}$

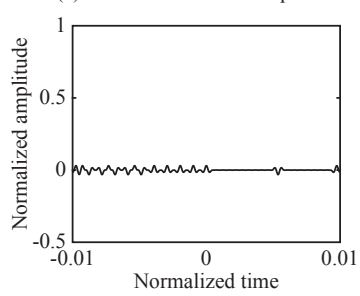

(b) Transmission of M-sequence B

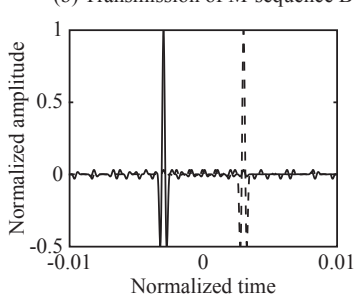

(c) Transmission of M-sequences A, B

Fig. 1 Correlation properties of sine waves modulated by preferred-pair M-sequences A and B. (a) Autocorrelation function of M-sequence A. (b) Cross-correlation function of M-sequence $\mathrm{B}$ with $\mathrm{M}$-sequence $\mathrm{A}$. (c) Cross-correlation functions of summed M-sequences $\mathrm{A}$ and $\mathrm{B}$ with each $\mathrm{M}$-sequence $\mathrm{A}$ or $\mathrm{B}$.

order M-sequence, interchannel interference of the preferred-pair M-sequences seems to be less of an issue.

A schematic of the proposed method of M-sequence pulse compression for a moving object is illustrated in Fig. 2. When the M-sequence-coded signal is Dopplershifted, the cycle length, which is the product of the signal duration in the binary character and $L$, is also expanded or compressed. In the autocorrelation function of the cyclic $\mathrm{M}$-sequence-coded signal, there is a peak at the time delay that corresponds to the cycle length. Therefore, the Doppler shift of the received cyclic $\mathrm{M}$-sequence-coded signal can be estimated from the peak in the autocorrelation function of the received signal [16]. Then, the reference signal is also Doppler-shifted to correlate with the Doppler-shifted $\mathrm{M}$-sequence-coded signal. Positions of non-Doppler objects are measured by cross-correlation of the received signal and the original reference signal. Meanwhile, positions of the moving object are measured by crosscorrelation of the received signal and the Doppler-shifted reference signal, which is expanded or compressed by the estimated Doppler velocity of the object.

\subsection{Experimental Setup}

The experimental setup for position and velocity measurements is illustrated in Fig. 3. The measurement system was composed of three microphones and two loudspeakers. The positions ( $x$ position, $y$ position) of microphones 1, 2, and 3 were $(600 \mathrm{~mm}, 230 \mathrm{~mm})$, $(900 \mathrm{~mm}, 230 \mathrm{~mm})$, and $(1,200 \mathrm{~mm}, 230 \mathrm{~mm})$, those of loudspeakers 1 and 2 were $(300 \mathrm{~mm}, 240 \mathrm{~mm})$ and $(1,500 \mathrm{~mm}, 240 \mathrm{~mm})$. The object was a stainless-steel pole whose diameter is $25 \mathrm{~mm}$. The pole moved at a constant velocity on the linear-motion stage. The speed of the stage was set at 240 or $360 \mathrm{~mm} / \mathrm{s}$, and the direction of motions was set at $147^{\circ}$ or $-132^{\circ}$. When the pole goes through a specific start point, the transmission of ultrasound is started. The start points were at $(980 \mathrm{~mm}, 1,320 \mathrm{~mm})$ and $(1,240 \mathrm{~mm}, 2,090 \mathrm{~mm})$. The carrier frequency of the Msequence-coded signal was $33.333 \mathrm{kHz}$. Therefore, the original cycle length was $61.41 \mathrm{~ms}$. Ultrasound coded by three cycles of $\mathrm{M}$-sequences $\mathrm{A}$ and $\mathrm{B}$ were transmitted

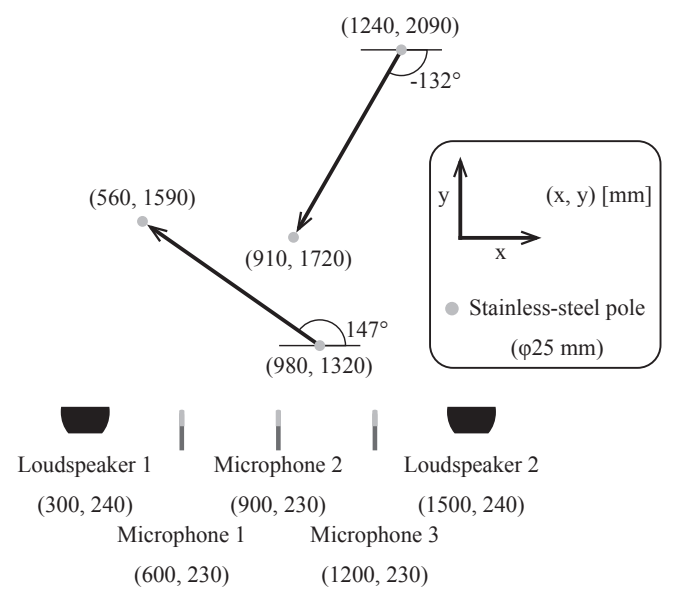

Fig. 3 Experimental setup for position and velocity measurements by multichannel $\mathrm{M}$-sequence pulse compression.
Fig. 2 Schematic of the proposed method of M-sequence pulse compression for a moving object. 
from loudspeakers 1 and 2, respectively. In these experiments, the kind of preferred-pair M-sequences does not seem to affect position and velocity measurements. Therefore, arbitrary preferred-pair M-sequences were selected. The number of feedback taps in LFSRs for M-sequences $\mathrm{A}$ and $\mathrm{B}$ are 1 and 3, respectively. In the case of $\mathrm{M}$ sequence $\mathrm{A}$, the position of the tap is between the 9th and 10th shift registers. In the case of $\mathrm{M}$-sequence $\mathrm{B}$, the positions are between the 6th and 7th, the 8th and 9th, and the 10th and 11th shift registers. Initial values in shift registers are both (00000000001). The interval of ultrasound transmission was $0.2 \mathrm{~s}$. Received signals were recorded at a sampling frequency of $2 \mathrm{MHz}$.

In this study, experiments of two situations, individual transmission (IT1, IT2) and simultaneous transmission (ST) of preferred-pair M-sequences, were conducted. In all transmissions, first, a moving target indication (MTI) filter was applied to the received signal for only Doppler velocity estimation. In the MTI filtering, the previous received signal was subtracted from the present received signal to remove non-Doppler components, that is, directly propagated ultrasound and echoes from stationary objects.

In the individual transmission from loudspeaker 1 or 2 (IT1, IT2), the Doppler velocity of the pole was estimated from the autocorrelation function of the MTI-filtered received signal in each microphone. The maximum peak in the autocorrelation function was selected within $\pm 10 \%$ of the original cycle length, that is, from 55.27 to $67.55 \mathrm{~ms}$. Then, the received signal without the MTI filter was correlated with each Doppler-shifted reference signal. In each cross-correlation function, there are three correlation peaks obtained by three-cycle transmission. To avoid the effect of truncation noise in M-sequence pulse compression, only echoes corresponding to the second M-sequence are considered [21]. Therefore, each cross-correlation function after $61.41 \mathrm{~ms}$ is used for subsequent processes. The acoustic image was formed from three cross-correlation functions by the synthetic aperture focusing technique in IT1 and IT2. For comparison with the simultaneous transmission, the acoustic image was also formed from three cross-correlation functions in IT1 and those in IT2 (IT). The pixel pitch of the acoustic image was $0.1 \mathrm{~mm}$. The position of the pole was determined as the position of the maximum brightness pixel on the image.

On the other hand, there are two Doppler components associated with each loudspeaker in the simultaneous transmission (ST). Therefore, two Doppler velocities of the pole were estimated from the autocorrelation function of the MTI-filtered received signal in each microphone. The maximum peak and the secondary peak, which is larger than sidelobes of the maximum peak, were determined within $\pm 10 \%$ of the original cycle length. In the case of the motion direction of $147^{\circ}$, the lower Doppler velocity was assigned to loudspeaker 1 . In the case of the motion direction of $-132^{\circ}$, the higher Doppler velocity was assigned to loudspeaker 1. Then, other Doppler velocities were assigned to loudspeaker 2 . When the secondary peak cannot be determined, the Doppler velocity estimated from the maximum peak is assigned to both loudspeakers. The received signal in each microphone was correlated with Doppler-shifted reference signals A and B. The processes of acoustic image formation and position determination were the same as the IT processes.

\section{RESULT}

Measurements were repeated 10 times at each angle and each speed for IT1, IT2, and ST. In the case of the motion direction of $147^{\circ}$, the mean values of estimated Doppler velocities at stage speed of 240 and $360 \mathrm{~mm} / \mathrm{s}$ are illustrated in Figs. 4 and 5. Moreover, errors, i.e., the differences from theoretical Doppler velocities, are also shown in Figs. 4 and 5. Theoretical Doppler velocities are calculated from the set stage speed and theoretical positions not considering actual positions on the pole surface where ultrasound reflected. At both speeds, there are similar trends of errors whether transmission is IT1,

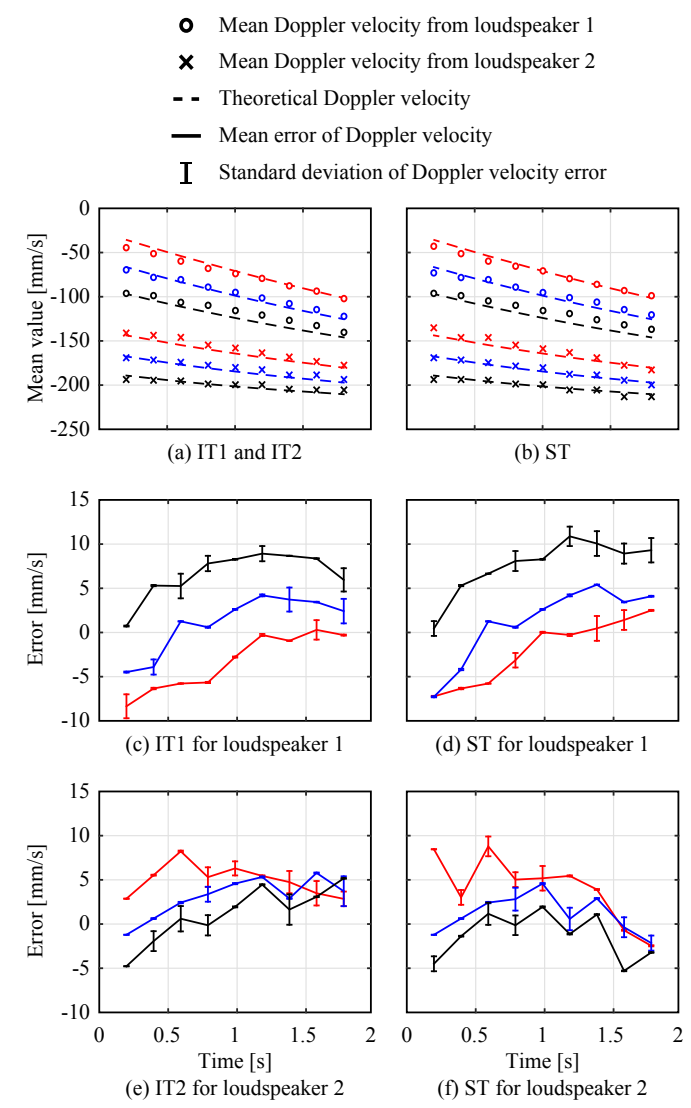

Fig. 4 Estimated Doppler velocities when the direction and the speed of the stage were respectively $147^{\circ}$ and $240 \mathrm{~mm} / \mathrm{s}$. Red: microphone 1, Blue: microphone 2, Black: microphone 3 . 
- Mean Doppler velocity from loudspeaker 1

X Mean Doppler velocity from loudspeaker 2

- - Theoretical Doppler velocity

- Mean error of Doppler velocity

I Standard deviation of Doppler velocity error

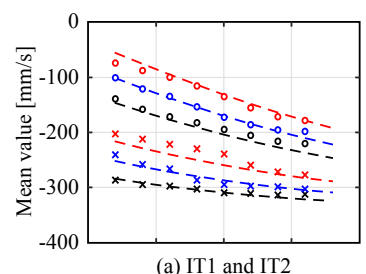

(a) IT1 and IT2

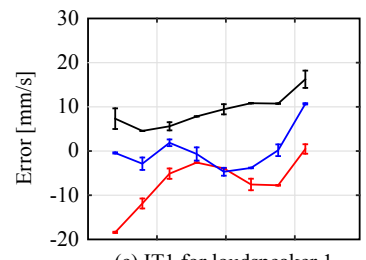

(c) IT1 for loudspeaker 1

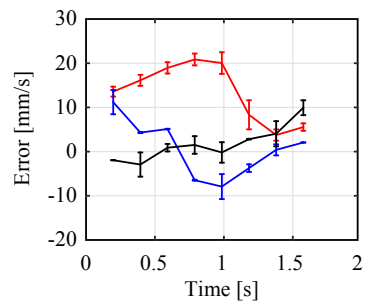

(e) IT2 for loudspeaker 2

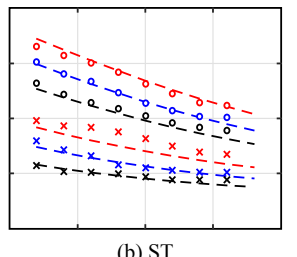

(b) ST

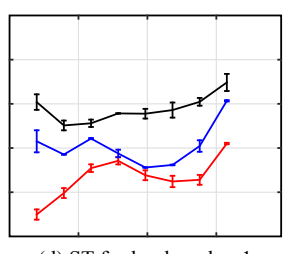

(d) ST for loudspeaker 1

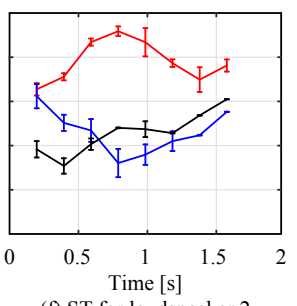

(f) ST for loudspeaker 2

Fig. 5 Estimated Doppler velocities when the direction and the speed of the stage were respectively $147^{\circ}$ and $360 \mathrm{~mm} / \mathrm{s}$. Red: microphone 1, Blue: microphone 2, Black: microphone 3.

IT2, or ST. Therefore, interchannel interference seems not to affect the accuracy of Doppler velocity estimation in these situations. In the case of loudspeaker 1, the estimated Doppler velocities were smaller at the beginning of the motion. Then, they became larger at the end. There are not such errors in the case of loudspeaker 2. Therefore, these errors of Doppler velocities seem to be geometric position errors. Furthermore, there also seem to be errors due to differences in actual reflection positions and environmental noise. In the case of the motion direction of $-132^{\circ}$, the mean values of estimated Doppler velocities and their errors when stage speeds were 240 and $360 \mathrm{~mm} / \mathrm{s}$ are illustrated in Figs. 6 and 7. There are similar trends, except for loudspeaker 1 at $240 \mathrm{~mm} / \mathrm{s}$ and ST. In this case, two Doppler velocities could not be estimated, that is, the same Doppler velocities were employed to loudspeaker 1 and loudspeaker 2. Therefore, only errors of Doppler velocities to loudspeaker 1 increased. In other situations, the estimated Doppler velocities of the pole were within $\pm 20 \mathrm{~mm} / \mathrm{s}$.

Determined positions of the pole are compared with paths of the pole in IT1, IT2, IT, and ST. To obtain each theoretical pole path, the pole was intermittently moved by

- Mean Doppler velocity from loudspeaker 1

X Mean Doppler velocity from loudspeaker 2

- - Theoretical Doppler velocity

- Mean error of Doppler velocity

I Standard deviation of Doppler velocity error
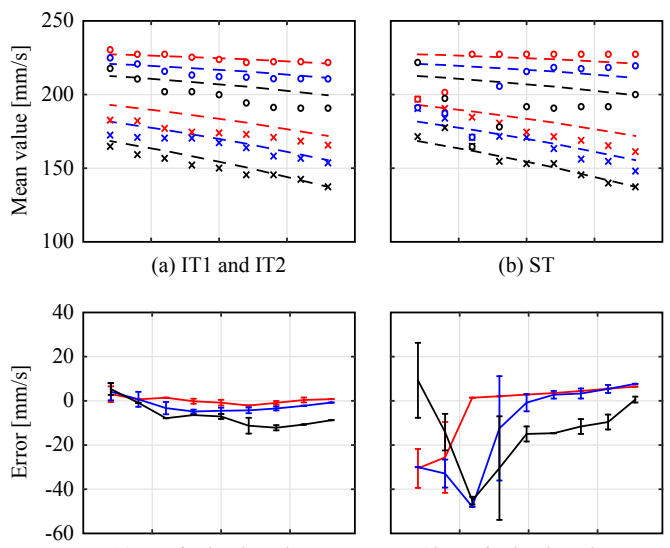

(c) IT1 for loudspeaker 1

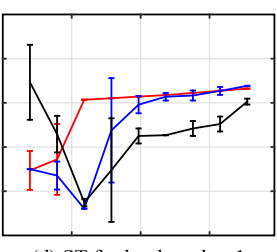

(d) ST for loudspeaker 1
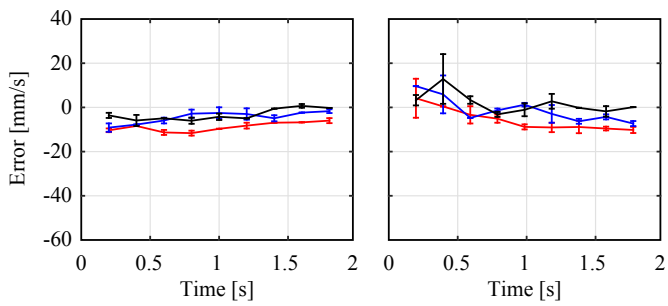

(e) IT2 for loudspeaker 2

(f) ST for loudspeaker 2

Fig. 6 Estimated Doppler velocities when the direction and the speed of the stage were respectively $-132^{\circ}$ and $240 \mathrm{~mm} / \mathrm{s}$. Red: microphone 1, Blue: microphone 2, Black: microphone 3 .

moving the stage. Then, 10 positions of the stationary pole between start points and end points were measured by the same processes (10 times at each position) in IT1, IT2, IT, and ST. Next, each path going through the 100 measured positions was drawn by the least squares method. Errors of positions are defined as distances from each pole path. In the case of the motion direction of $147^{\circ}$, errors of determined positions were within $2 \mathrm{~mm}$, as illustrated in Fig. 8. In the case of the motion direction of $-132^{\circ}$, errors of determined positions were larger in the beginning with $240 \mathrm{~mm} / \mathrm{s}$ of IT1 and ST, as illustrated in Fig. 9.

\section{DISCUSSION}

In Doppler velocity estimation of ST, two Doppler velocities could not be estimated in some situations. In autocorrelation functions of IT1 and IT2, there are peaks that indicate Doppler-shifted cycle lengths, as illustrated in Figs. 10 and 11. The autocorrelation function of ST corresponds to the sum of those of IT1 and IT2. When the peak interval of IT1 and IT2 are relatively large and the peak of IT1 and the dip of IT2 or vice versa don't overlap, each peak can be identified in the autocorrelation function of ST, as shown in Fig. 10. In other cases, two peaks 
- Mean Doppler velocity from loudspeaker 1

X Mean Doppler velocity from loudspeaker 2

- - Theoretical Doppler velocity

- Mean error of Doppler velocity

I Standard deviation of Doppler velocity error
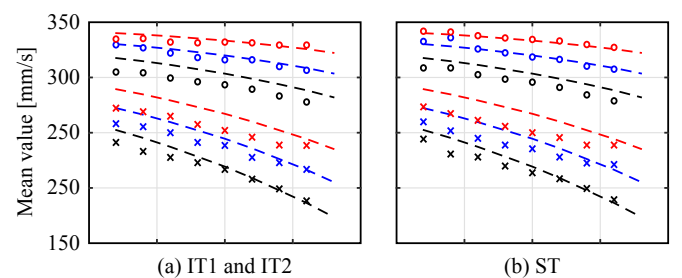

(b) ST

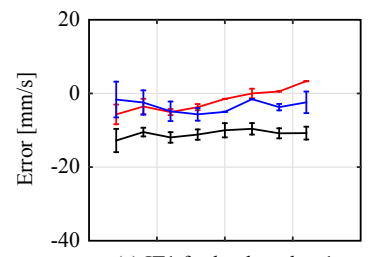

(c) IT1 for loudspeaker 1
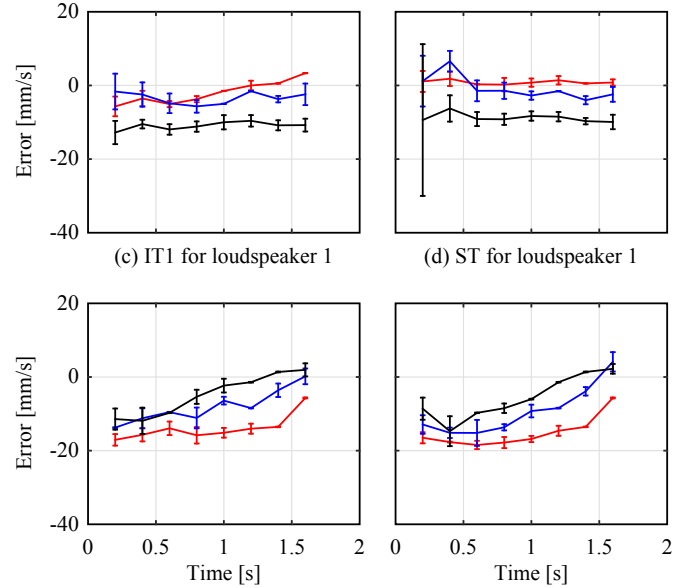

(e) IT2 for loudspeaker 2

(f) ST for loudspeaker 2

Fig. 7 Estimated Doppler velocities when the direction and the speed of the stage were respectively $-132^{\circ}$ and $360 \mathrm{~mm} / \mathrm{s}$. Red: microphone 1, Blue: microphone 2, Black: microphone 3.

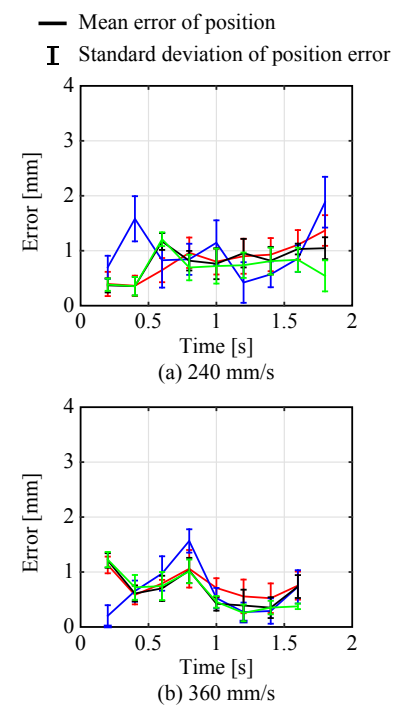

Fig. 8 Errors of determined positions from the pole path when the direction of the stage was $147^{\circ}$. Red: IT1, Blue: IT2, Black: IT, Green: ST.

cannot be identified, as shown in Fig. 11. In the proposed method of position and velocity measurements by simultaneous transmission of multichannel M-sequences, there
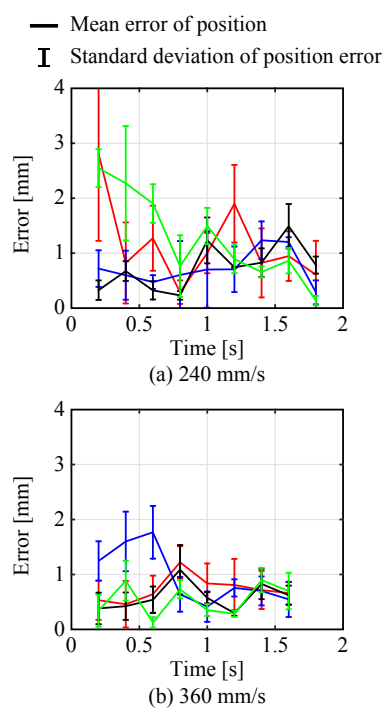

Fig. 9 Errors of determined positions from the pole path when the direction of the stage was $-132^{\circ}$. Red: IT1, Blue: IT2, Black: IT, Green: ST.

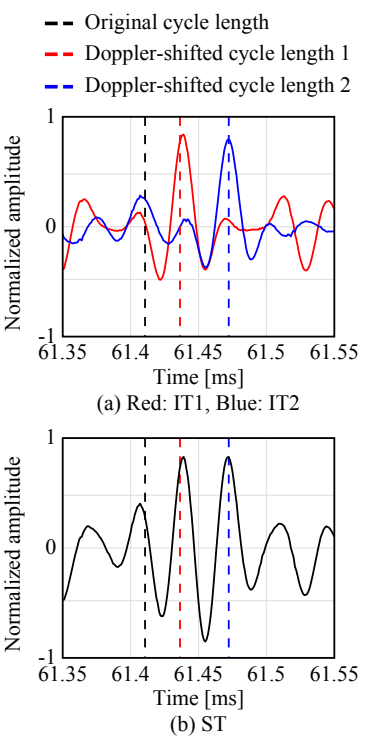

Fig. 10 Autocorrelation functions indicating cycle lengths when the direction and the speed of the stage were respectively $147^{\circ}$ and $240 \mathrm{~mm} / \mathrm{s}$.

are some situations in which Doppler velocities cannot be estimated.

Multichannel M-sequence pulse compression is employed to improve the spatial resolution of an acoustic image. Acoustic images of first measurements in IT1, IT2, and ST when the direction and the speed of the stage were respectively $-132^{\circ}$ and $240 \mathrm{~mm} / \mathrm{s}$ are illustrated in Fig. 12. The resolution of an acoustic image is generally defined as the number of brighter pixels, e.g., whose brightness are more than 0.5 , around the maximum brightness pixel. Positions of bright regions in IT1 and IT2 are different because ultrasound from each loudspeaker 


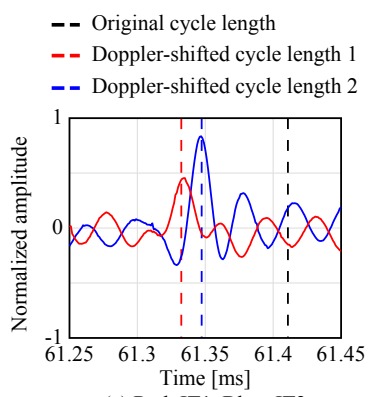

(a) Red: IT1, Blue: IT2

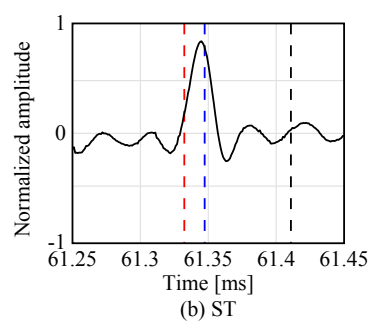

Fig. 11 Autocorrelation functions indicating cycle lengths when the direction and the speed of the stage were respectively $-132^{\circ}$ and $240 \mathrm{~mm} / \mathrm{s}$.
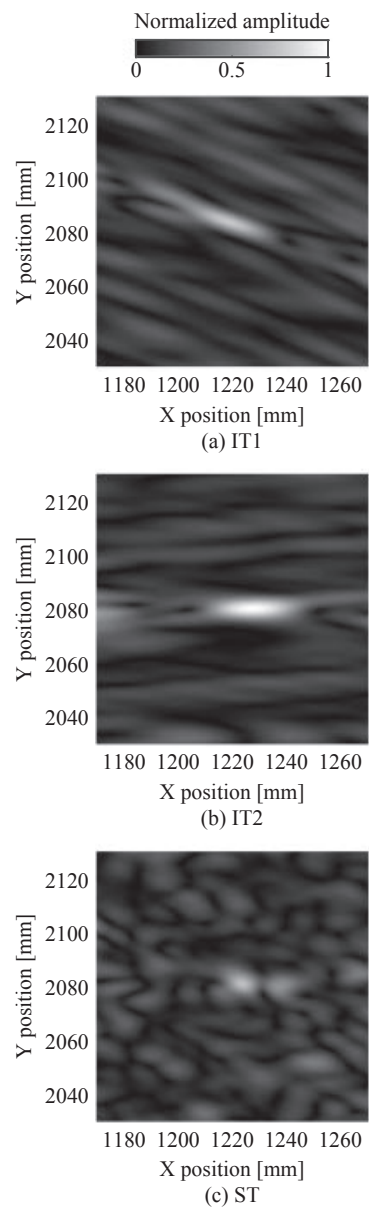

Fig. 12 Acoustic images of first measurements when the direction and the speed of the stage were respectively $-132^{\circ}$ and $240 \mathrm{~mm} / \mathrm{s}$.

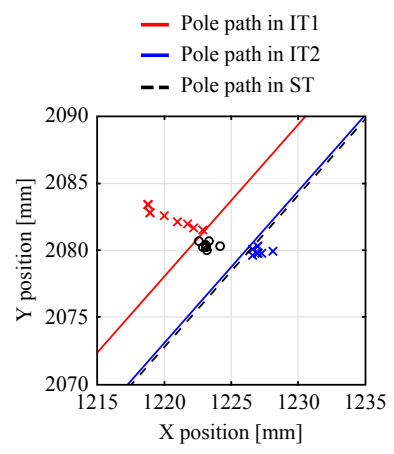

Fig. 13 Determined positions of first measurements when the direction and the speed of the stage were respectively $-132^{\circ}$ and $240 \mathrm{~mm} / \mathrm{s}$.

reflect at the different positions on the pole surface. Therefore, the resolution in ST is improved because the bright region in ST is overlap of regions in IT1 and IT2.

The determined positions in IT1, IT2, and ST are also different. In this situation, errors of determined positions were larger in IT1 and ST. Therefore, all positions determined 10 times in each transmission are illustrated in Fig. 13. The determined positions in IT1, IT2, and ST are distributed around bright regions in Fig. 12, respectively. Therefore, larger position errors in IT1 or ST seem to be caused by shifts of maximum brightness pixels in each region. Shapes and magnitudes of these bright regions vary depending on amplitudes and phases in 3 (IT1 and IT2) or 6 (ST) cross-correlation functions, which are also associated with the accuracy of Doppler velocity estimation.

\section{CONCLUSION}

In this study, Doppler velocities and positions of a moving pole of $25 \mathrm{~mm}$ diameter were measured by pulse compression in the simultaneous transmission of preferredpair M-sequences in two-dimensional experiments. In the proposed method of Doppler velocity estimation using the autocorrelation function of the received signal, Dopplershifted cycle lengths of M-sequences are selected. In some situations, however, accurate Doppler velocities could not be estimated when the peaks in the autocorrelation function corresponding to Doppler-shifted cycle lengths were overlapped. In other situations, Doppler velocities could be estimated within $\pm 20 \mathrm{~mm} / \mathrm{s}$ of the theoretical values. In the proposed method, furthermore, positions of the pole are determined from the maximum brightness pixel in acoustic images. The resolution, that is, the number of pixels whose brightness are more than 0.5 , in acoustic images was improved by the transmission of $\mathrm{M}$-sequences from two loudspeakers. Errors of determined positions of the pole were within $2 \mathrm{~mm}$ in most situations. When the position of maximum brightness pixel in acoustic images 
is shifted because of inaccurate Doppler velocities, the errors were larger than $2 \mathrm{~mm}$. However, the position errors increased as a result of interchannel interference were within $4 \mathrm{~mm}$.

In this work, acoustic images were formed and positions of targets were detected assuming that objects are similar to point reflectors and the number of targets is small. However, there are many objects, whose scale are larger than the wavelength of ultrasound, such as a human being. Furthermore, the objects may have specular surfaces, such as a building pillar. Therefore, methods of image formation or target detection and classification must be improved to advance future work.

\section{REFERENCES}

[1] B. S. Lim, S. L. Keoh and V. L. L. Thing, "Autonomous vehicle ultrasonic sensor vulnerability and impact assessment," Proc. 2018 IEEE 4th World Forum on The Internet of Things, Singapore, pp. 231-236 (2018).

[2] W. Xu, C. Yan, W. Jia, X. Ji and J. Liu, "Analyzing and enhancing the security of ultrasonic sensors for autonomous vehicles," IEEE IoT-J, 5, 5015-5029 (2018).

[3] P. Krishnan, "Design of collision detection system for smart car using Li-Fi and ultrasonic sensor," IEEE Veh. Technol., 67, 11420-11426 (2018).

[4] S. Hirata, Q. Sun, M. Ueda and H. Hachiya, "Measurement of road surfaces by reflection characteristics of airborne ultrasound," Acoust. Sci. \& Tech., 37, 322-325 (2016).

[5] F. Lam and J. Szilard, "Pulse compression techniques in ultrasonic non-destructive testing," Ultrasonics, 14, 111-114 (1976).

[6] J. J. Kroszczynski, "Pulse compression by means of linearperiod modulation," Proc. IEEE, 57, 1260-1266 (1969).

[7] S. Hirata, M. K. Kurosawa and T. Katagiri, "Accuracy and resolution of ultrasonic distance measurement with high-timeresolution cross-correlation function obtained by single-bit signal processing," Acoust. Sci. \& Tech., 30, 429-438 (2009).

[8] S. Hirata and M. K. Kurosawa, "Ultrasonic distance and velocity measurement using a pair of LPM signals for crosscorrelation method: Improvement of Doppler-shift compensation and examination of Doppler velocity estimation," Ultrasonics, 52, 873-879 (2012).
[9] N. Thong-un, S. Hirata, Y. Orino and M. K. Kurosawa, "A linearization-based method of simultaneous position and velocity measurement using ultrasonic waves," Sens. Actuator A Phys., 233, 480-499 (2015).

[10] P. J. Bendick and V. L. Newhouse, "Ultrasonic random-signal flow measurement system," J. Acoust. Soc. Am., 56, 860-865 (1974).

[11] S. Ohtsuki and M. Okujima, "Ultrasonic Doppler velocity meter by double M-sequence modulation method," Proc. 2nd World Congress on Ultrasonics in Medicine, Rotterdam (1973).

[12] R. C. Waag, J. B. Myklebust, W. L. Rhoads and R. Gramiak, "Instrumentation for noninvasive cardiac chamber flow rate measurement," Proc. 1972 IEEE Ultrasonics Symp., Boston, pp. 74-77 (1972).

[13] B. B. Lee and E. S. Furgason, "High-speed digital Golay code flaw detection system," Proc. 1981 IEEE Ultrason. Symp., Chicago, pp. 888-891 (1981).

[14] T. G. Birdsall, "Acoustic telemetry for ocean acoustic tomography," IEEE J. Ocean. Eng., OE-9, 237-241 (1984).

[15] R. A. Altes and D. P. Skinner, "Sonar velocity resolution with a linear-period-modulated pulse," J. Acoust. Soc. Am., 61, 1019-1030 (1977).

[16] S. Hirata and H. Hachiya, "Doppler velocity estimation based on spectral characteristics of M-sequence-modulated signals in ultrasonic measurement for moving objects," Jpn. J. Appl. Phys., 52(7S), 07LC03 (2013).

[17] Y. Ikari, S. Hirata and H. Hachiya, "Ultrasonic position and velocity measurement for a moving object by M-sequence pulse compression using Doppler velocity estimation by spectrum-pattern analysis," Jpn. J. Appl. Phys., 54(7S1), 07HC14 (2015).

[18] C. B. Burckhardt, P. A. Grandchamp and H. Hoffmann, “An experimental $2 \mathrm{MHz}$ synthetic aperture sonar system intended for medical use," IEEE Trans. Sonics Ultrason., SU-21(1), 1-6 (1974).

[19] R. Gold, "Optimal binary sequences for spread spectrum multiplexing,” IEEE Trans. Inf. Theory, 13, 619-621 (1967).

[20] K. Yamanaka, S. Hirata and H. Hachiya, "Evaluation of correlation property of linear-frequency-modulated signals coded by maximum-length sequences," Jpn. J. Appl. Phys., 55(7S1), 07KC09 (2016).

[21] S. Hirata and H. Hachiya, "Truncation-noise characteristics of finite-length M-sequence,” Acoust. Sci. \& Tech., 36, 254-261 (2015). 